\title{
Application of solid-state storage in big data processing of products traceability
}

\author{
Zuliang Wang ${ }^{1}$, Lingyan Fan ${ }^{2}$, Robin Zhou ${ }^{3}$, Libin Wang ${ }^{4}$ \\ ${ }^{1}$ Information Engineering College, Xijing University, Shanxi Xian 710123, China \\ ${ }^{2}$ Microelectronics Research Institute, Hangzhou Dianzi University, Huangzhou 310018, China \\ ${ }^{3}$ Sage Microelectronics Corporation, Hangzhou, 311215, China \\ ${ }^{4}$ Beijing Space Information Relay and Transmision Technology Research Center, Bejing, 100081, \\ China
}

Keywords: Agricultural products traceability; Big data processing; Solid-state storage

\begin{abstract}
Traceability system of agricultural products can effectively let the whole process of agricultural production be monitored. It can realize that the flow of products can be traced back, product quality information can be query and the product can be recalled. Solid-state storage technology has the advantages of less power consumption and strong seismic resistance. Therefore, the application of solid-state memory in the traceability of agricultural products will bring high efficiency and reliable storage means. In this paper, the application of solid-state storage in the big data of agricultural products being studied, we design the tracking and tracing system flow char, the three-layer structure model and a control system for solid state storage.
\end{abstract}

\section{Introduction}

With the rapid development of information technology, big data has been applied in various industries, including agriculture. The integration of big data into the traceability of agricultural products is an effective choice. Big data + traceability of agricultural products is the big data technology used in the cultivation of agricultural products, processing, transportation, storage and transportation, sales, information management and distribution of a series of modern agricultural products circulation. It collects, stores, manages and analyzes the mass data produced by each link, to achieve the whole process of production of agricultural products can be monitored, the flow of products can be traced back, product quality information can be found and the problem can be recalled products.

The traceability system of agricultural products requires all kinds of agricultural information to be collected all the time, and the amount of super data has exceeded the traditional database management ability. This application has a high demand for the processing and analysis of large data. The storage medium is the key technology in large data processing and analysis. Only supported by the high reliability, safe storage technology, big data analysis and processing can be achieved. Solid-state memory stores data by storing the switching state of the transistors on the chip. Solid state memory has the advantages of low power consumption and strong shock resistance because it has no read-write head and does not need to rotate. Therefore, the application of solid-state memory in the traceability of agricultural products will bring high efficiency and reliable storage means, which provides strong protection for agricultural products traceability system. In this paper, the application of solid-state storage in the big data of agricultural products being studied, we design the tracking and tracing system flow char, the three-layer structure model and a control system for solid state storage.

\section{Solid-State Storage Medium in Large Data Processing of Agricultural Products Traceability}

The storage medium of big data in agricultural products traceability not only needs large storage space, but also must be a kind of quick access, erasing times enough, non-volatile storage medium with power-off protection function. At present, there are several kinds of solid state storage media 
that meet this requirement.

FRAM, also known as ferroelectric memory, is the core of ferroelectric crystal material. FRAM combines the advantages of ROM and the advantages of RAM, such as nonvolatile data storage characteristics, unlimited read and write, high-speed read and write, and low power consumption.

Flash Memory, also known as flash memory, is a nonvolatile solid-state storage medium. Its storage characteristics are equivalent to the hard disk, and can keep the data for a long time without current supply.

NV-SRAM is the abbreviation of Non-volatile SRAM, which is SRAM. NV-SRAM uses SRAM+EEPROM mode, the realizing of the battery without the need for non-volatile storage, chip interface, read and write timing and the standard SRAM fully compatible. NV-SRAM operations are usually performed in SRAM. Only when the outside of the sudden power failures, the data are stored into the EEPROM. When the system is powered on, it will automatically copy the data in EEPROM to SRAM. The removable storage medium includes mobile hard disk, SD/MMC card, U disk, CF card, etc.

Big data processing in agricultural products traceability require large storage medium capacity and high reliability. Neither FRAM nor NV-SRAM alone can meet the requirements. Combined with the power consumption and cost of the system, we decided to use FRAM+NAND Flash solid-state storage scheme in the traceability system of agricultural products traceability.

\section{Design of Agricultural Products Traceability System}

Agricultural product quality traceability and tracking includes two aspects. Tracking is the ability to follow a particular unit or group of products from the upstream to the downstream of the supply chain. Traceability is the ability to identify a particular unit or a group of products from the downstream to the upstream of the supply chain, that is, the ability to trace the production, breeding, production, processing and other aspects of a farm product by tracing back code. Tracking and tracing system flow chart is shown in Figure. 1.

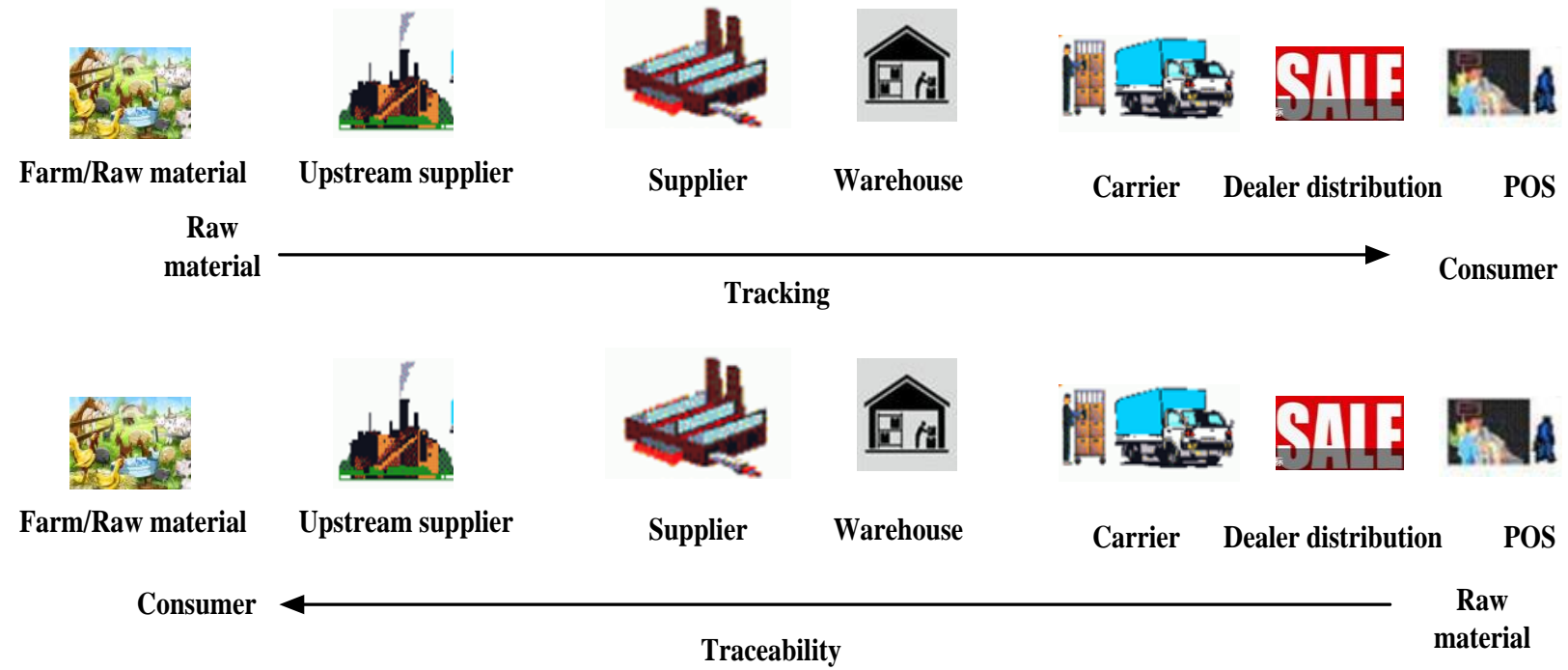

Figure 1. Tracking and tracing system flow char

In order to realize the quality of agricultural products traceability and tracking, three-layer structure model is established. It is shown in Figure. 2.

Personalized customized service is used for the cultivation, acquisition, processing, circulation, marketing and other aspects of personalized information customization. Therefore, the tracing system can better adapt to a variety of complex production processes, information collection, processing and query, statistics, including the dictionary structure, query information, regulatory information customization, etc.. Traceability information collection system is used for the whole collection of the bottom of the chain information, basic data management, including the dictionary maintenance management, information collection, data upload, etc.. 


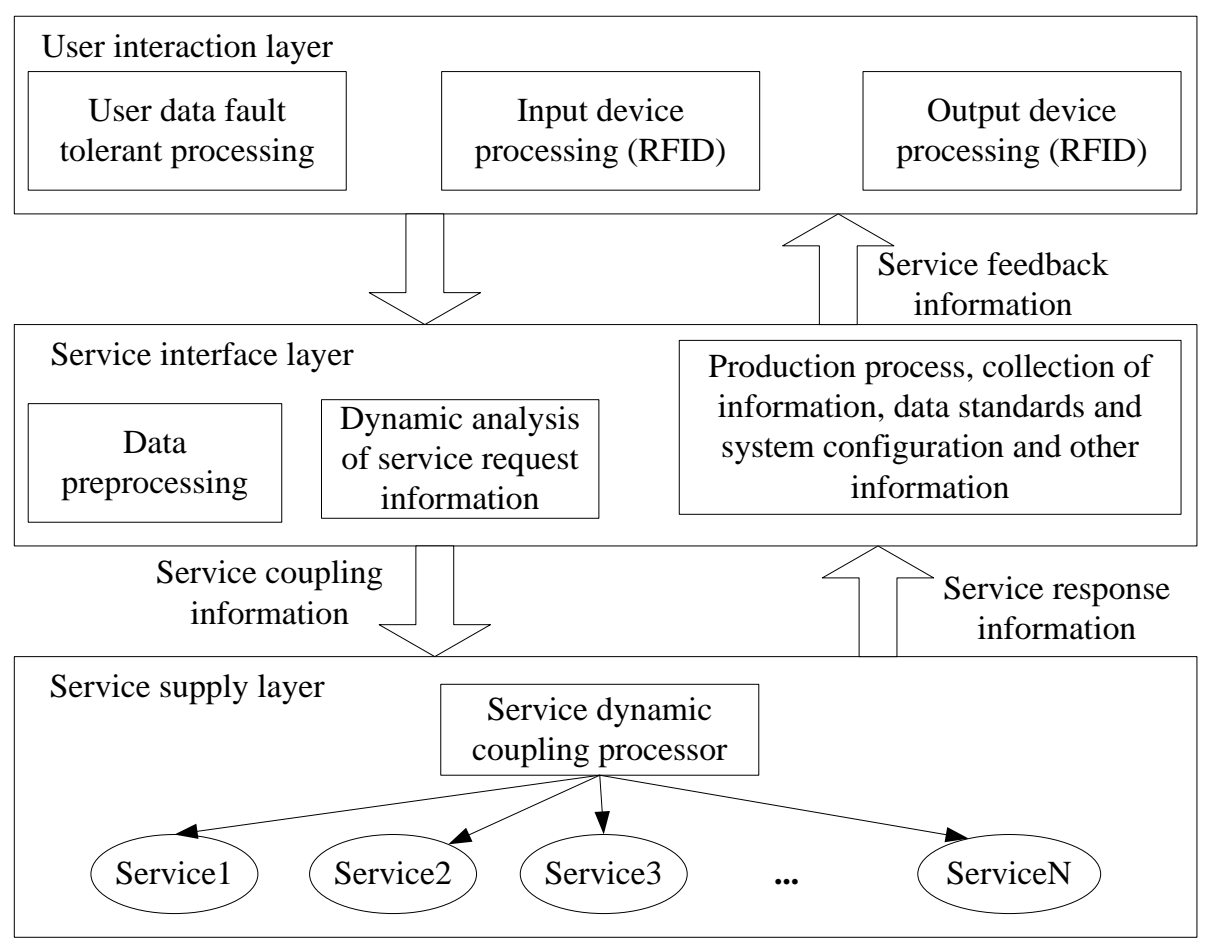

Figure 2. Three-layer structure model

Data collection system to receive enterprise data, summary, data management, query analysis, resulting in a retrospective chain, data package upload, etc.. Data preprocessing is the completion of the enterprise data preprocessing, including data cleaning, data integration, data transformation, data reduction.

\section{Solid State Storage Schemes in Big Data Processing}

Large data processing solid state storage scheme uses the FRAM+NAND Flash to achieve, has the characteristics such as fast FRAM speed, low power consumption, strong stability and NAND Flash capacity. Among them, the intermediary FRAM as the data transfer, when it needs to save the data in the subtle level speed, the multiple flashes FRAM is used to storage. Over a certain period of time or when a power failure occurs, immediately transfer data from FRAM to NAND Flash, So as to avoid the phenomenon of data loss due to power down. Therefore, the scheme meets the requirements of high reliability, high speed of access, and high reliability of power failure protection function.

The big data processing system uses the high performance FM24CL04 ferroelectric memory and the K9K1G08U0M serial flash memory to work together. The FM24L256 operating voltage is 5V, working current is $150 \mathrm{uA}$, the storage density is $4 \mathrm{Kbit}$, the write operation is up to the bus speed, no write extended time, having excellent read and write life which can reach 10 billion times, using $\mathrm{I} 2 \mathrm{C}$ bus interface.

The internal function of the K9K1G08U0M is shown in figure 3. The working voltage of K9K1G08U0M is 3.3V, 8 bit data bus, each Page has 528 Byte, 32 Page forms a Block. A K9K1G08U0M chip contains a total of 8192 Block, Its total capacity is 528 Byte $\times 32$ Page $\times 8192$ Block = 132 MByte.K9K1G08U0M's first Block guaranteed by the manufacturer number is its accuracy, through different command sequences to achieve the whole page read, write, block erase, multi-page read, write back and other data operations. 


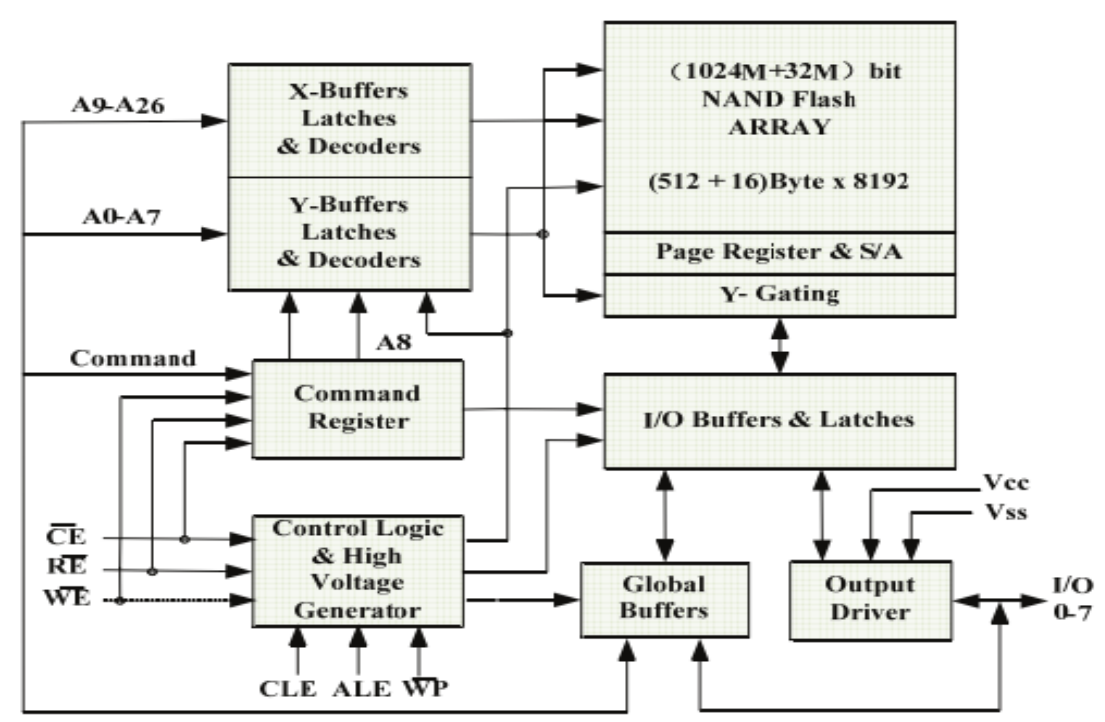

Figure 3. Function diagram of K9K1G08U0M

\section{Summary}

In this paper, the application of solid-state storage in the large data processing of agricultural products traceability is studied. The integration of big data into the traceability of agricultural products is an effective choice. The application model of agricultural products traceability and the three layer architecture based on large data processing are designed.

\section{Acknowledgements}

This work is supported by the Industrial Science and Technology Project of Shaanxi Province (Grant No. 2016GY-141), Zhejiang Key Laboratory of Solid State Drive and Data security(Grant No.2015E10003), and the Scientific Research Fund Project of Xijing University(Grant No.XJ160140).

\section{References}

[1] Wagner, G. L. \&Glassheim. E. Traceability of Agricultural Products[J]. Film Watch Divi sion Marketing Plan. 2003. 5:1-17.

[2] Sahin. E.,Dallery. Y., Gershwin. S., Performance evaluation of a traceability system. Proceedings of IEEE International Conference on Systems[J]. Man and Cybernetics. 2002,25(3): 210-218.

[3] Regattieri A, Gamberi M, Manzini R. Traceability of food products: General framework and experimental evidence[ J] .Journal of Food Engineering 2007,81(2): 347-356.

[4] Gantz J, Reinsel D. The digital universe in 2020: Big Data, Bigger Digital Shad-ows, and Biggest Growth in the Far East [R]. 2013.

[5] Hilbert M, Lopez P. The World's Technological Capacity to Store, Communicate, and Compute Information [J]. Science. 2011, 332: 60-65.

[6] IBM. The Square Kilometer Array: The Ultimate Big Data Challenge [R]. 2013.

[7] Biology M V. The big challenges of big data [J]. Nature. 2013, 498: 255-260.

[8] Wen Chuan Yang, He Chen,Qing Yi Qu. Research of a MapReduce Model to Process the Traffic Big Data[J] . Applied Mechanics and Materials . 2014 (548).

[9] Chi Yang,Xuyun Zhang,Changmin Zhong,Chang. A spatiotemporal compression based approach for efficient big data processing on cloud[J] . Journal of Computer and System Sciences . 2014.

[10]Maharana P R. Reducing writes to solid state drive cache memories of storage controllers[J]. 2015. 